Botelho LS, Arboit EL \& Freitag VL. (2020). Nurses' performance in the care of the prevention and treatment of pressure injuries. Research, Society and Development. 9(7):1-19, e775974644.

\title{
Atuação do enfermeiro no cuidado a prevenção e tratamento de lesões por pressão
}

Nurses' performance in the care of the prevention and treatment of pressure injuries

Desempeño de las enfermeras en el cuidado de la prevención y el tratamiento de las lesiones por presión

Recebido: 18/05/2020 | Revisado: 24/05/2020 | Aceito: 26/05/2020 | Publicado: 12/06/2020

\section{Luciane dos Santos Botelho}

ORCID: https://orcid.org/0000-0002-2968-5715

Universidade de Cruz Alta, Brasil E-mail:negalu08011985@hotmail.com

Éder Luís Arboit

ORCID: https://orcid.org /0000-0001-8929-5228

Universidade de Cruz Alta, Brasil

E-mail: earboit@unicruz.edu.br

Vera Lúcia Freitag

ORCID: https://orcid.org/0000-0002-5897-7012

Universidade de Cruz Alta, Brasil

E-mail: verafreitag@hotmail.com

\section{Resumo}

Objetivo: conhecer a atuação de enfermeiros nas ações de prevenção e tratamento de lesões por pressão, no ambiente hospitalar. Metodologia: estudo de abordagem qualitativa do tipo descritivo e exploratório. Participaram do estudo 12 enfermeiros de um hospital do interior gaúcho. A coleta de dados ocorreu em setembro de 2018, por meio da entrevista semiestruturada, sendo os dados analisados pela Analise de Conteúdo Temático. Resultados: evidencia-se a utilização de estratégias de cuidado com foco na prevenção: avaliação da pele, medidas para diminuir a pressão sobre proeminências ósseas e cuidados higiênicos. Quanto ao tratamento destaca-se: uso de coberturas específicas conforme características da lesão, hidratação e suporte nutricional, aliado aos encontros de educação premente. Conclusão: a atuação do enfermeiro no cuidado aos pacientes com lesões está pautada na prevenção e tratamento, utilizando tecnologias inovadoras de cuidado, proporcionando uma assistência 
segura, diminuindo o tempo de internação e possibilitando uma melhora na qualidade de vida e saúde do paciente.

Palavras-chave: Enfermagem; Prevenção; Tratamento; Lesão por pressão.

\begin{abstract}
Objective: to know the role of nurses in the prevention and treatment of pressure injuries in the hospital environment. Methodology: a qualitative study of the descriptive and exploratory type. Twelve nurses from a hospital in the interior of Rio Grande do Sul participated in the study. Data collection took place in September 2018, through the semi-structured interview, with the data analyzed by Thematic Content Analysis. Results: there is evidence of the use of care strategies with a focus on prevention: skin assessment, measures to reduce pressure on bone prominences and hygienic care. Regarding the treatment, the following stand out: use of specific coverings according to the characteristics of the lesion, hydration and nutritional support, combined with pressing education meetings. Conclusion: the role of nurses in caring for patients with injuries is based on prevention and treatment, using innovative care technologies, providing safe care, reducing hospital stay and enabling an improvement in the patient's quality of life and health.
\end{abstract}

Keywords: Nursing; Prevention; Treatment; Pressure injury.

\title{
Resumen
}

Objetivo: conocer el papel de las enfermeras en la prevención y el tratamiento de las lesiones por presión en el entorno hospitalario. Metodología: estudio cualitativo de tipo descriptivo y exploratorio. Doce enfermeras de un hospital en el interior de Rio Grande do Sul participaron en el estudio. La recopilación de datos tuvo lugar en septiembre de 2018, a través de la entrevista semiestructurada, con los datos analizados por Análisis de contenido temático. Resultados: existe evidencia del uso de estrategias de atención con un enfoque en prevención: evaluación de la piel, medidas para reducir la presión sobre las prominencias óseas y cuidado higiénico. En cuanto al tratamiento, destacan los siguientes: uso de recubrimientos específicos según las características de la lesión, hidratación y soporte nutricional, combinado con reuniones educativas urgentes. Conclusión: el papel de las enfermeras en el cuidado de pacientes con lesiones se basa en la prevención y el tratamiento, utilizando tecnologías de atención innovadoras, brindando atención segura, reduciendo la estancia hospitalaria y permitiendo una mejora en la calidad de vida y salud del paciente.

Palabras clave: Enfermería; Prevención; Tratamiento; Lesión por presión. 


\section{Introdução}

As lesões por pressão (LPP) constituem um dos principais eventos adversos potencialmente evitáveis que ocorrem nos serviços de saúde. Trazem como consequências, há o aumento dos gastos públicos e a diminuição da qualidade de vida do paciente (Silva et al., 2017). Nesta direção a LPP é caracterizada como um dano localizado na pele e/ou tecidos subjacentes, geralmente sobre uma proeminência óssea, resultante da pressão isolada ou combinada com forças de cisalhamento e/ou fricção, como também pelo microclima, nutrição, perfusão e condições do tecido (Npuap, 2018).

O desenvolvimento de LPP em pacientes hospitalizados vem sendo considerado um marcador na qualidade da atenção à saúde, como também na segurança do paciente, tanto na atenção primária, quanto na atenção especializada. Deste modo impõe-se como um desafio aos profissionais de saúde, gestores e mais ainda para os próprios usuários/pacientes e seus respectivos familiares/cuidadores. Neste contexto, ressalta-se a importância do cuidado multi e interdisciplinar a fim de que se possa assistir o usuário/paciente sempre se levando em conta a sua individualidade.

Estima-se que a ocorrência de LPP está entre 4 a 16\% de pacientes hospitalizados em países desenvolvidos (Brasil, 2017). O maior número é evidenciado nas Unidades de Terapia Intensiva (UTI). Estudo concluiu que a prevalência variou entre $35,2 \%$ a 63,6\% e a incidência entre $11,1 \%$ e $64,3 \%$ (Vasconcelos \& Caliri, 2017). Em outro estudo, evidenciou-se que a prevalência destas lesões varia entre 3 e $66 \%$, sendo fator influenciador as condições do paciente, a doença e o tipo de instituição onde o paciente está cuidado. Os autores destacam que no ambiente hospitalar, ela varia de 5 a $40 \%$. No que tange a idosos internados, correspondem a taxa de $18,8 \%$ e, em pacientes de terapia intensiva, $17,7 \%$ a 35,2\% (Campos et al. 2017).

Sua ocorrência está associada não somente a falhas no cuidado, como também a fatores extrínsecos (pressão, fricção; cisalhamento e unidade) e intrínsecos (idade avançada, com diminuição progressiva das atividades habituais, nutrição prejudicada, perfusão tecidual diminuída, uso de medicamentos, uso e/ou abuso de álcool e tabaco, e alterações glicêmicas e dos níveis pressóricos). A presença de comorbidades associadas, como doenças de origem pulmonar, infecciosa, neurológica, renal, neoplasia, cardiopatia e depressão também podem desencadear o surgimento ou agravamento das LPP (Rocha et al., 2020).

Autores como Serrano et al., (2017) acrescentam como fatores preditivos do desenvolvimento LPP o tempo de permanência na UTI, tempo de ventilação mecânica (VM), 
(CC BY 4.0) | ISSN 2525-3409 | DOI: http://dx.doi.org/10.33448/rsd-v9i7.4644

hemodiálise intermitente ou hemofiltração veneno-venosa contínua, suporte vasopressor uso de sedação. Além destes, estudo italiano revela como fator decisivo para o desenvolvimento de LPP, aqueles pacientes em falência múltipla de órgãos e que apresentam escores baixos na escala de Braden (Lucchini et al., 2018).

Destaca-se que LPP pode apresentar-se estágios distintos: o estágio 1 apresenta pele íntegra com eritema que não embranquece; o estágio 2 caracteriza-se por perda da pele em sua espessura parcial com exposição da derme; o estágio 3 é caracterizado por perda da pele em sua espessura total; e o estágio 4, por perda da pele em sua espessura total e perda tissular. Além disso, é possível que uma LPP seja do tipo não classificável - quando apresenta perda da pele em sua espessura total e perda tissular não visível - ou do tipo LPP tissular profunda quando apresenta descoloração vermelho escura, marrom ou púrpura, persistente, e que não embranquece (Npuap, 2018).

Diante do exposto, o enfermeiro, juntamente com demais membros da equipe de enfermagem desempenham atividades consideradas essenciais na prevenção e tratamento das LPP. No que tange aos aspectos preventivos Brasil (2017) destaca a avaliação criteriosa da pele mantendo-a limpa e seca, uso de colchão especial, almofadas e/ou de coxins para redistribuir a pressão em áreas de proeminências ósseas e manutenção da higiene corporal. Além destes, acrescenta-se a hidratação constante da pele, posicionamento adequado no leito, alimentação rica em vitaminas e proteínas aos primeiros sinais de LPP, e mudanças de decúbito a cada duas horas (Frazão et al.; 2019).

Por outro lado, o tratamento precisa estar centrado no uso de coberturas que possam absorver o exsudato e promover a melhora da reepitelização celular. Assim, a prescrição das coberturas devera levar em conta aspectos como: tipo de tecido, estadiamento da lesão, quantidade de exsudato, presença de sangramento, sensibilidade à dor e presença de infecção. Dentre as coberturas incluem-se: as primárias, as secundárias e os dispositivos de fixação/suporte (Hc-Ufmt/Ebserh, 2018).

Neste contexto, os enfermeiros são responsáveis pelo cuidado direto e contínuo de pacientes com LPP. Além disso, ele precisa estar atento às mudanças e/ou adequações impostas pelo quadro clinico de cada paciente, subsidiando novas opções de prevenção e tratamento, as quais estão devidamente regulamentas pela Resolução 567/2018, do Conselho Federal de Enfermagem. Deste modo, possibilitando o cuidado da equipe de enfermagem mais efetivo (Figueira, Backes \& Knihs, 2018). Diante do exposto, este estudo tem como objetivo: conhecer a atuação de enfermeiros nas ações de prevenção e tratamento de lesões por pressão, no ambiente hospitalar. 


\section{Metodologia}

Estudo de abordagem qualitativa do tipo descritiva e exploratória como preconiza Pereira et al., (2018). Entende-se que os estudos qualitativos propiciam ao pesquisador o entendimento mais aprofundado sobre a forma como determinadas situações ocorrem (Cooper \& Schindler, 2016). A referida pesquisa teve como cenário um hospital de médio porte de caráter privado situado em um município do Sul do Brasil.

Participaram do estudo 12 enfermeiros de acordo com os critérios de inclusão: ocupar cargo de enfermeiro, tempo de trabalho de pelo menos seis meses e desenvolver atividades nas unidades de clínica médica, clínica cirúrgica e terapia intensiva. Dentre os critérios de exclusão estão: os profissionais que tiverem afastados por licenças de qualquer natureza ou férias durante o período de coleta dos dados. A coleta de dados ocorreu no período de setembro de 2018, após aprovação do Comitê de Ética e Pesquisa, sob o parecer n ${ }^{\circ} 2.869 .098$ e Certificado de Apresentação para Apreciação Ética (CAAE) no 95106418.7.0000.5322.

Aos participantes foi garantido o anonimato, sigilo das informações e a possibilidade de desistência em qualquer fase da pesquisa, sem qualquer prejuízo ou penalização. Foram explicados em linguagem acessível, a justificativa, os objetivos e os procedimentos utilizados na pesquisa e também o destino das informações e a ausência de benefícios diretos ou dispêndios decorrentes de sua participação na pesquisa. Como instrumento utilizou-se a entrevista semiestruturada, contendo questões abertas e fechadas referentes ao objeto de estudo. Permite ao entrevistado contribuir no processo de investigação com liberdade e espontaneidade, sem perder a objetividade (Moré, 2015).

Nas entrevistas buscou-se informações referentes à caracterização dos participantes e aqules relacionados ao objetivo do estudo: como ocorre o cuidado de enfermagem ao paciente com lesão por pressão no ambiente hospitalar? As entrevistas foram gravadas com auxílio de um gravador digital, com duração média de 15 minutos. O encerramento amostral se deu quando o objetivo da pesquisa foi alcançado, levando-se em consideração o critério de saturação de dados (Minayo, 2017).

Os participantes assinaram o Termo de Consentimento Livre e Esclarecido em duas vias, ficando uma via com este e outra com o pesquisador. Foram identificados pela letra inicial da categoria profissional e sequencialmente numerado de acordo com a sequência. Exemplo: Enfermeiro 1 (E1). Para a análise dos dados, utilizou-se a técnica da Análise Temática (Minayo, 2014). Prevê três fases: pré-análise, exploração do material e o tratamento dos resultados obtidos e interpretação. 


\section{Resultados}

Foram entrevistados 12 enfermeiros atuantes nas áreas de clínica médica, cirúrgica, e terapia intensiva, sendo 11 do sexo feminino e um integrante do sexo masculino. O intervalo etário variou de 29 e 57 anos de idade. O tempo de atuação varia de 1.5 e 29 anos. Quatro dos participantes possuem título de especialistas e seis estão cursando pós-graduação.

As informações obtidas junto aos participantes do estudo foram classificadas conforme a análise de Minayo (2014). Assim, foi possível agrupar os resultados em uma categoria temática que trata sobre: "Estratégias de cuidado utilizadas por enfermeiros na prevenção e tratamento de lesão por pressão".

\subsection{Estratégias utilizadas por enfermeiros na prevenção de lesão por pressão}

No cuidado ao paciente com LPP, o enfermeiro desenvolve várias ações aliado a equipe multiprofissional e multidisciplinar, principalmente o acompanhamento no que tange a prevenção, a qual é fundamental para o cuidado do paciente, como se observa a seguir:

Mudança de decúbito, reposicionamento, utilizando o passante para transferir o paciente de um leito para a maca. Todos os pacientem utilizam o colchão piramidal elou pneumáticos, além de coxins, ácidos graxos, hidratação de pele hidratação via oral e suporte nutricional. Além disso, realizamos treinamentos com os trabalhadores e orientamos os familiares e cuidadores, utilizamos curativos de proteção como hidrocolóides e filme transparente (E1).

Colchão piramidal, rotina de mudança de decúbito e reposicionamento, conforme prescrição de enfermagem. Medidas higiênicas como banho de leito e higiene íntima assistidos e realizado pela equipe de enfermagem. Curativos para prevenção como filmes, hidrocoloides, creme barreira, curativo com silicone. Uso de ácidos graxos essências, creme barreira, orientação quanto ao uso de hidratantes (E11).

Evidencia-se que a instituição preconiza a prevenção de LPP, uma vez que disponibiliza produtos para esta finalidade. Nesta direção, o enfermeiro e demais membros da 
(CC BY 4.0) | ISSN 2525-3409 | DOI: http://dx.doi.org/10.33448/rsd-v9i7.4644

equipe de enfermagem precisam estar familiarizados com o processo que envolve o desenvolvimento de uma lesão. Atentar para as questões importantes como prevenção, quanto na recuperação, a exemplo de estratégias de cuidado que são de competência do enfermeiro:

Sempre se tenta cada vez mais buscar meios e produtos antes de tudo para prevenção do aparecimento (E4).

[...] onde eu trabalho é oferecido quase tudo para prevenção. Temos vários produtos $e$ modos de estimular o paciente, familiares e técnicos de enfermagem a prevenir $e$ tratar lesões por pressão, como relógio para mudança de decúbito, creme barreira, filme transparente e demais produtos (E5).

Todavia, existem fragilidades no cuidado ao paciente com LPP. Alguns familiares apresentam resistência à mudança de decúbito no período noturno, uma vez que referem medo do paciente não dormir mais. Outras vezes essa mudança de decúbito é dificultada em pacientes que apresentam obesidade.

[...] Decúbito dorsal necessário e solicitado pelo médico quando o paciente se encontra no respirador (ventilação mecânica) (E1).

Há algumas dificuldades quanto à resistência dos familiares com a mudança de decúbito, pois muitas vezes os pacientes estão dormindo e eles não querem que acorde. Pedem para deixa-lo quieto, mas devemos insistir para que seja feita a mudança sempre orientando sobre a importância (E3).

Observa-se que várias estratégias de prevenção, como: relógio para mudança de decúbito, creme barreira, filme transparente e demais produtos, além de maneiras para estimular o paciente, familiares e técnicos de enfermagem a prevenir as LPP. Todavia, ressalta-se que cabe ao enfermeiro avaliar a pele do paciente e prescrever cuidado adequado. É importante que o enfermeiro perceba que essas competências fazem parte do seu cotidiano, realizando as orientações e supervisionando, conforme descreve o profissional enfermeiro: 
Através das medidas preventivas e conscientes no cuidado, sendo a prevenção o mais adequado (E2).

Em relação às fragilidades, quanto à resistência dos familiares, pode-se refletir em relação ao conhecimento que os cuidadores/familiares têm a este respeito. É um processo que faz parte do cuidado de enfermagem, sensibilizar o cuidador quanto aos riscos, atentando para a necessidade desta estratégia de prevenção e cuidado.

\subsection{Ações de enfermagem no tratamento ao paciente com lesão por pressão}

O enfermeiro tem papel fundamental na assistência ao paciente com LPP, visto ser ele quem prescreve o cuidado, levando em conta a necessidade específica de cada paciente. Avalia a pele, acompanha a evolução diariamente, registrando e discutindo os aspectos relacionados com as características de cada lesão e do próprio paciente junto à equipe.

A assistência se dá com um cuidado geral envolvendo todos da equipe acompanhando a evolução da lesão. Também cabe a nós acompanhar pra ver se o produto utilizado e os demais cuidados estão adequados. Daí reforço a questão como higiene, mudança de decúbito, manter pele seca, sempre que possível hidratada, avaliando alimentação, exames laboratoriais, ingesta líquida, demais cuidados (E7).

A assistência de enfermagem e criteriosa e minuciosa. Avaliamos a pele, através da escala de Braden, realizamos desbridamentos, prescrevemos cuidados específicos para cada paciente, reforço com colaboradores a importância de seguir os cuidados prescritos, bem como comunicar alteração. Acompanhamos a evolução diariamente, com fotos e registros adequados e o grupo discutindo o caso (E9).

A assistência se dá com um cuidado geral envolvendo todos da equipe acompanhando a lesão, a evolução se o produto utilizado, e os demais cuidados como higiene, mudança de decúbito, manter pele seca, sempre que possível hidratada, avaliando alimentação, exames laboratoriais, ingesta líquida, demais cuidados. Aqui a gente utilizar a escala de Bradem que é um instrumento importante na avaliação das condições da pele do paciente. (E10). 
Diante do exposto nos relatos dos participantes, percebe-se que está entre as atribuições do enfermeiro, a avaliação criteriosa da condição clinica de cada paciente, o que lhe exige preparo técnico científico. Com os avanços tecnológicos, a aquisição de vários novos produtos e técnicas fazem parte do cotidiano do ser/fazer enfermeiro. Como se pode observar nos depoimentos a seguir:

Aqui nós temos disponíveis as coberturas como filmes, hidrocolóide simples e creme barreira, para o tratamento temos também alguns hidrogeis (E7).

Para tratamento temos hidrocoloide com alginato, hidrogel com alginato, hidrogel com age, placa de carvão, compressa com emulsão de petrolatum, baiatam, phmb gel de limpeza e iodosorb (E3).

Todavia, ressaltam a importância do cuidado integral, visto que pacientes mais debilitados tem maior propensão a desenvolver uma lesão por pressão, e percebem a importância da ênfase na prevenção em detrimento ao tratamento. Todavia, quando o paciente é acometido por uma LPP, tem-se uma equipe qualificada, bem como produtos de qualidade para tratamento desta forma:

O que posso perceber é que o mais fácil é sempre o tratamento, mas é necessário dar ênfase a prevenção [...] (E7).

[...] temos uma equipe qualificada e produtos com adequada função para melhorar a cicatrização da lesão (E10).

Outras ações que fazem parte do cuidado de enfermagem relacionado à LPP, além dos cuidados preventivos supracitados, com ênfase aos pacientes de maior risco, são os são os momentos de Educação Permanente (EP) em serviço. Nestes espaços, são discutidos os casos e a elaboração de planos de ações para o tratamento:

Sim, recebemos treinamentos mensais e nos reunimos no grupo de feridas NEPELE (Núcleo de Lesões de Pele), com reuniões quinzenais, para discussões de lesões, e alternativas no tratamento. Falamos sobre os sinais e sintomas, estágios da lesão, maneiras de preveni-las e o tratamento em sí (E3). 
Diante dos relatos, observa-se que a Educação Permanente (EP) em serviço constituise como ferramenta essencial a fim de instrumentalizar os enfermeiros nas ações de cuidado ao paciente com LPP, uma vez que propicia a ele a identificação da sintomatologia, estadiamento, formas de prevenção e o tratamento.

\section{Discussão}

Evidenciou-se que os participantes utilizam estratégias para a prevenção de LPP: mudança de decúbito, reposicionamento, utilização do passante para transferir o paciente de um leito para a maca, uso de coxins e colchão piramidal e/ou pneumáticos para todos os pacientes. No cuidado á pele, medidas higiênicas como banho de leito e higiene íntima, assistidas e realizadas pela equipe de enfermagem, hidratação com o uso de ácidos graxos essenciais e creme barreira, curativos de proteção para prevenção como filmes transparentes, hidrocolóides e curativo com silicone. Além disso, a instituição faz uso da educação permanente como ferramenta para realizar orientações e treinamentos aos profissionais, pacientes e familiares/cuidadores.

Nesta perspectiva, o momento do banho de leito, é essencial para avaliação da pele do paciente por parte do enfermeiro, bem como a hidratação com o uso de emulsões como ácidos graxos e creme barreira para o cuidado com a pele e proeminências ósseas, fazendo uma barreira de proteção à pele. É o momento em que o profissional de enfermagem tem a oportunidade de conhecer e observar o paciente, favorecendo a realização de um exame físico minucioso nas proeminências ósseas identificando possíveis alterações na pele.

Estudo destaca a importância dos cuidados de prevenção de LPP, mencionando a mudança de decúbito a cada duas horas, reposicionamento, uso de coxins, colchões especiais, inspeção diária da pele, no momento do banho e nas higienes íntimas, sendo a equipe de saúde capacitada para realizar tais ações. A hidratação da pele também é fundamental no cuidado preventivo e suporte nutricional (Neiva, et al., 2019).

Nesse contexto, destaca-se a relevância do conhecimento cientifico da equipe de enfermagem na prevenção de LPP, principalmente ao paciente que tem um grau avançado de desidratação, e pouca ou nenhuma mobilidade. O conhecimento proporciona a autonomia, visto que a enfermagem, nesta premissa, necessita reformular protocolos de enfermagem no qual se avalia os fatores de riscos, avaliação da integridade a pele, em como atentar para os fatores extrínsecos, como, umidade, fricção e cisalhamento. 
Além disso, propõe a avaliação dos fatores de riscos, cuidados com a pele e tratamento precoce, com mudanças de decúbito frequente e o uso de suporte que reduzam a pressão, hidratação constante da pele. Ressalta-se que o exame físico minucioso no momento do banho configura-se como uma importante ferramenta orientadora da prática do enfermeiro, o qual contará com um subsídio teórico para o desenvolvimento de uma assistência humanizada, efetiva e segura (Costa et al., 2018, Silva, et al., 2019), pois o conhecimento não basta apenas, é preciso aperfeiçoar habilidades e atitudes, que abarquem os três grandes eixos de competência - saber ,saber fazer e querer fazer (Campoi, et al., 2019).

Nesta premissa, os participantes destacam a realização de treinamentos com os profissionais. Assim, Rodrigues et al., (2018) mencionam que para ser promovida a prevenção de uma LPP é imprescindível à aquisição de competências em relação à temática por parte dos profissionais de saúde, sobretudo no que tange aos trabalhadores que fazem parte da equipe de enfermagem, os quais atendem os clientes de forma continuada e mais diretamente.

Neste sentido, a capacitação de profissionais se faz necessária, bem como a orientação aos familiares e cuidadores, considerando que a adoção de medidas preventivas para reduzir a incidência de LPP, é considerada um importante indicador de qualidade assistência. Assim, faz-se necessário dar ênfase a orientação aos pacientes, familiares e cuidadores sobre prevenção, causas e fatores de risco da LPP (Campoi, et al., 2019).

Destaca-se a importância da educação em saúde aos familiares, não somente para o cuidado hospitalar, mas pensando na alta do paciente, visto que em domicílio são os familiares que prestarão o cuidado direto ao paciente e, se este, estiver bem orientado poderá dispensar o cuidado ao paciente de maneira resolutiva, diminuindo as reinternações.

Outro fator importante na prevenção de LPP que deve ser levado em consideração é o suporte nutricional para hidratação oral. A privação nutricional e a ingestão nutricional inadequada são os principais fatores de risco para o desenvolvimento de LPP. Nesta premissa, a avaliação e o fornecimento de nutrição apropriada com base em diretrizes nutricionais baseadas em evidências devem ser considerados como um ingrediente essencial no cuidado a este paciente. Portanto, a triagem apropriada do estado nutricional, deve ter a colaboração com nutricionista habilidoso e a assim a administração adequada de fórmulas especializadas consistem em macro e micronutrientes (Saghaleini, et al., 2018).

Os participantes mencionaram que a ênfase de seu trabalho é a prevenção, buscando estratégias de cuidado que possibilitem prevenir o aparecimento de LPP. Destacam que a instituição disponibiliza os produtos necessários à prevenção, e a capacitação como estratégias de estimular o paciente, familiares e técnicos de enfermagem a prevenir e tratar 
lesões por pressão. Uma ferramenta de cuidado é o relógio para mudança de decúbito. Tratase de um impresso na cabeceira do leito e identificado com cores para diferenciar os horários. Assim, a equipe pode realizar a mudança de decúbito no horário preconizado, atendendo o princípio de prevenção de acordo com o protocolo (Sanches, et al., 2018).

Observa-se ainda que muitas vezes que, os procedimentos relacionados à mudança de decúbito podem ficar fragilizados, especialmente nos pacientes em VM. Alguns familiares resistem às mudanças de decúbito, quando o paciente está dormindo, para não acordá-lo. Todavia, a enfermagem compreende que neste contexto fazem-se necessárias orientações pertinentes com o objetivo de sensibilizar os familiares quanto à importância da prevenção de LPP, criando estratégias para que este cuidado seja efetivamente realizado, treinando, instrumentalizando o familiar/acompanhante para que possa auxiliar neste processo.

O uso de fármacos vasopressores predispõe o desenvolvimento de LPP (Silva, et al., 2014). Diante disso, acredita-se que a mudança de decúbito possa ser realizada em pacientes em VM, sem prejuízo para a resistência pulmonar e com vantagens em relação à prevenção de lesão por pressão.

Quanto ao cuidado dispensado ao paciente obeso, estudo aponta que gera sobrecarga especialmente nos cuidados de higiene, mudança de decúbito, pois precisaram ser realizados com três ou mais profissionais. Contudo, os pacientes obesos não geram necessariamente um aumento na carga de trabalho em relação à horas de trabalho, mas sim implica em maior quantitativo de profissionais, devido à dificuldade de mobilização no leito, demandando Sendo assim, o número reduzido de profissionais de enfermagem pode favorecer um aumento de eventos adversos (Goulart, et al., 2017), como LPP.

Diante disso, compreende-se que faz-se necessário um cuidado preventivo, utilizando de diversas estratégias a fim de evitar eventos adversos como uma LPP, visto que a instituição pesquisada oferece diversos recursos para prevenção, bem como capacitação cotidiana aos profissionais para o cuidado específico a cada paciente. Assim, faz-se necessário que a família também participe deste cuidado, e, para tanto, o enfermeiro tem papel importante no sentido de sensibilizar esta ou o acompanhante para fortalecer o cuidado preventivo, levando em consideração que prevenir uma LPP é mais adequado em detrimento ao tratamento, além de ser um importante indicador de qualidade.

Os participantes ressaltam que a assistência de enfermagem é criteriosa e minuciosa, ocorre envolvendo todos da equipe, acompanhando a evolução LPP diariamente com fotos, registros adequados e discussão de casos, no sentido de observar se o produto utilizado está adequado àquela lesão, reforçando a importância da higiene, mudança de decúbito, manter a 
pele seca, hidratada, avaliando alimentação, exames laboratoriais, ingesta líquida e demais cuidados. Ainda, avaliam a pele por meio da escala de Braden, realizam desbridamentos, prescrevem cuidados específicos para cada paciente, orientando sobre o seguimento destes, bem como comunicar qualquer alteração.

A avaliação de uma LPP deve ser realizada pelo enfermeiro, bem como o emprego do tratamento adequado, melhor cobertura de acordo com as características da lesão e acompanhamento da evolução (Torres, et al., 2018). Este cuidado do enfermeiro com o cliente com LPP requer muito além da prática do curativo, como por exemplo, conhecimento e compreensão da fisiologia da pele e da cicatrização, e mais importante o conhecimento científico sobre os tipos de coberturas existentes no mercado (Silva et al., 2017).

Dentre os cuidados de enfermagem, destaca-se a avaliação da pele por meio da Escala de Braden, a qual é um instrumento de avaliação do risco para LPP mais utilizado mundialmente, leva em consideração a fisiopatologia das LPP e avaliação de seis parâmetros importantes que são: percepção sensorial, umidade, mobilidade, atividade, nutrição, fricção e cisalhamento. Todavia, estes itens são determinados por uma pontuação que resultam em um total, denominado de score, o qual varia de 6 a 23 pontos e a classificação pode ser: risco muito alto - escore $\leq 9$, risco alto - 10 a 12 , risco moderado - 13 a 14, risco baixo - 15 a 18 e sem risco - 19 a 23 (Bergstrom, et al., 1987). Neste contexto, esta torna-se uma importante ferramenta, pois apresenta maior especificidade e sensibilidade para mensuração, confiança para identificar pacientes de alto risco que necessitam de cuidados adicionais para evitar eventos prejudiciais (Riccioni, et al., 2019).

Nesta premissa. Rodríguez-Acelas e Cañon-Montañez (2017) referem que as contribuições das escalas nos ambientes de cuidado clínico ajudam na avaliação e possibilitam a estratificação do risco. Para, além disso, os autores descrevem que o uso de escalas orienta a priorização das intervenções, acompanham a evolução do paciente, e assim, qualificam o processo de cuidar, otimizando tempo dos profissionais, além de trazer impacto positivo na segurança do paciente.

Diante dos relatos evidenciou-se que a instituição disponibiliza uma serie produtos, os quais são fundamentais para o processo de reepitelização celular. Dentre eles destaca-se: as coberturas como filmes, hidrocolóide simples, creme barreira, hidrocoloide com alginato, hidrogel com alginato, hidrogel com ácidos graxos, placa de carvão ativado, compressa com emulsão de petrolatum, curativo de espuma de poliuretano, gel de limpeza polihexanida e relógio para mudança de decúbito são eficazes na prevenção e tratamento de lesões por pressão, os quais são vistos como potencialidade do cuidar em enfermagem. 
Nesta perspectiva, a literatura revela que a utilização de produtos específicos para o tipo de LPP, corresponde ao chamado curativo perfeito, e este é o que mantém um ambiente fisiologicamente saudável no leito da lesão e contribui na diminuição do tempo de cicatrização. Este ambiente compreende: prevenção e controle da infecção, desbridamento dos tecidos inacessíveis, controle do exsudato, controle do odor e proteção da ferida, todavia, lesões com cavidades profundas, necessitam de atenção especial (Lobato, et al., 2017).

Observa-se pelas informações dos participantes que a instituição pesquisada disponibiliza produtos conforme as características de cada lesão, fato que facilita o tratamento e qualifica o cuidado. Ainda destaca-se que os profissionais recebem treinamentos mensais, além de encontros grupais com o NEPELE comissão de prevenção, tratamento e controle de LPP organizado pela instituição. Desenvolvem reuniões quinzenais, em que discutem assuntos referentes à LPP, bem como planejamento de alternativas de tratamento. Os assuntos tem enfoque nos sinais e sintomas, estágios da lesão, prevenção e tratamento.

Acredita-se que a formação de uma comissão supracitada, traz excelentes resultados. A exemplo desta instituição pesquisada, estudo que objetivou relatar a experiência de implantação da Comissão de Prevenção e Tratamento de Lesões na Pele em um hospital público, concluiu que o enfermeiro é o profissional de referência para esses cuidados, visto que ele é o protagonista das atividades, as quais trazem excelentes resultados, trabalhando na promoção, prevenção e tratamento das LPP, bem como minimizando o surgimento de lesões complexas. $\mathrm{O}$ atendimento é individualizado com foco em cada paciente e educação permanente das equipes assistenciais por meio de cursos específicos sobre desbridamentos, curativos e coberturas, como prevenir LPP, dentre outros (Torres, et al., 2018).

Torna-se fundamental a ênfase a prevenção em detrimento ao tratamento de LPP, visto que as LPP contribui para os elevados números de prevalência e incidência a nível mundial, uma vez que são de difícil cicatrização, e são associadas à comorbilidades e complicações que dependendo do paciente, relacionado ao histórico clinico pregresso, podem se tornar fatais (Sousa, et al., 2017). Neste contexto, os programas de treinamento/capacitação são fundamentais, assim como o aperfeiçoamento destes e sua avaliação para o desenvolvimento do cuidado de enfermagem, não somente relacionados à LPP, visando impulsionar transformações nas instituições de saúde dentro de uma visão crítica e alicerçada na realidade, resultante na construção de conhecimento para a organização, para o profissional da área e para a sociedade, trazendo benefícios e melhorias (Aroldi; Perez; Mira, 2018).

No desenvolvimento de uma LPP, o tempo de internação é maior, aumentando os gastos e talvez prejudicando na recuperação desse paciente, por isso se torna indispensável à 
prevenção, com ela a instituição tem menores gastos e desgaste emocional ao paciente e aos familiares/cuidadores, tornando a permanência hospitalar em um curto prazo. Uma LPP estagio avançado torna-se potencialmente mais complicada.

Sendo Assim, a prevenção é uma importante aliada da segurança do paciente, trazendo benefícios ao paciente, à instituição e aos profissionais. Todavia, no tratamento, a disponibilidade de coberturas apropriadas às características da lesão, bem como a comissão NEPELE, a qual fornece apoio por meio de capacitação aos profissionais, qualifica o trabalho, com foco no cuidado específico para cada paciente.

\section{Considerações Finais}

Evidenciou-se que a atuação de enfermeiros nas ações de prevenção e tratamento de lesões por pressão, no ambiente hospitalar. Identificou-se que o enfermeiro desenvolve seu trabalho com foco na prevenção. Por meio da classificação de risco de cada paciente, pela escala de Braden desde a admissão hospitalar até a alta. $\mathrm{O}$ enfermeiro avalia as condições da pele do paciente a fim de prescrever o tratamento, seja este preventivo ou prescrevendo o tipo de cobertura ideal para aquele cuidado de acordo com as características desta.

Observa-se que as estratégias de prevenção utilizadas pelos enfermeiros consistem em: mudança de decúbito, reposicionamento, utilização do passante para transferir o paciente de um leito para a maca, uso de coxins e colchão piramidal e/ou pneumáticos para todos os pacientes. No cuidado á pele, medidas higiênicas como banho de leito e higiene íntima, assistidas e realizadas pela equipe de enfermagem, hidratação com o uso de ácidos graxos essenciais e creme barreira, curativos de proteção para prevenção como filmes transparentes.

Em relação ao tratamento utiliza-se: cremes de barreira, gel de limpeza polihexanida, hidrogel com alginato, hidrogel com ácidos graxos, curativo hidrocoloide, curativo de espuma de poliuretano, hidrocoloide com alginato, placa de carvão ativado e compressa com emulsão de petrolatum. Além disso, destaca-se a hidratação e nutrição do paciente como fatores que influenciam para a prevenção das lesões, auxiliando na cicatrização. Os encontros de educação permanente contribuem para um olhar mais atento do enfermeiro e demais membros da equipe de enfermagem, instigando a reflexão sobre as melhores práticas de cuidado.

Estudos como este podem contribuir para um olhar mais atento para a prevenção e tratamento das LPP, com a utilização de tecnologias de cuidado apropriadas a cada tipo de lesão. O estudo apresenta limitações no sentido de que os participantes foram apenas enfermeiros de um único hospital, situado na região noroeste do Rio Grande do Sul/Brasil. 
Instituição esta de caráter privado que dispõe vários recursos para prevenção e tratamento de LPP. Assim, sugere-se que desenvolvam-se outras pesquisas em diferentes cenários, qualificando assim a assistência ao paciente.

\section{Referências}

Aroldi J. B. C, Peres H. H. C, Mira V. L (2018). Impact perception at work from an online training on the prevention of pressure injury. Texto \& Contexto - Enfermagem. 27(3): e3020016.

Bergstrom $\mathrm{N}$ et al. (1987). The Braden Scale for predicting pressure sore risk. Nurs Res. 36(4): 205-10.

Brasil. (2017). Nota Técnica GVIMS / GGTES Nº 03/2017 Práticas seguras para prevenção de Lesão por Pressão em serviços de. Saúde. Agência Nacional de Vigilância Sanitária Anvisa.

Campoi A. L. M et al (2019). Permanent education for good practices in the prevention of pressure injury: almost-experiment. Revista brasileira de enfermagem. 72(6): 1646-52.

Campos, SF et al. (2010). Fatores associados ao desenvolvimento de úlceras de pressão: o impacto da nutrição. Rev Nutr. 23(5): 703-14.

Conselho Federal de Enfermagem - Cofen (2018). Resolução n. 567 de 29 de janeiro de 2018. Regulamenta a atuação da Equipe de Enfermagem no Cuidado aos pacientes com feridas. Brasília.

Cooper D. R \& Schindler P. S. Métodos de pesquisa em administração. 12. ed. Porto Alegre: AMGH Editora Ltda, 2016.

Costa G. S et al (2018). Bed bath in critical care patients: an integrative review. Rev baiana de enferm. 32(1): e20483. 
Figueira T. M, Backes M. T. S \& Knihs N. S. (2018). Elaboração de um guia de cuidados de enfermagem para tratamento de pacientes com lesões por pressão. Revista de Pesquisa: Cuidado é Fundamental Online. 10(3): 322-326.

Frazão J. M et al (2019). A Abordagem do enfermeiro na prevenção de feridas em pacientes hospitalizados. Revista Enfermagem Atual In Derme. 88(26): 1-1.

Goulart L. L et al. (2017). Carga de trabalho de enfermagem relacionada ao índice de massa corporal de pacientes críticos. Acta Paul Enferm. 30(1): 31-38.

HC-UFTM/Ebserh - Hospital de Clínicas da Universidade Federal do Triângulo Mineiro (HCUFTM). (2018). Prevenção e tratamento de lesão por pressão. Serviço de Educação em Enfermagem. Uberaba-MG.

Lobato C. P et al. (2017). Tele condutas - Lesão por pressão. Núcleo de saúde da Universidade Federal do Rio Grande do Sul.

Lucchini A et al (2018). Incidenza e fattori di rischio associati allo sviluppo di lesioni da pressione in una terapia intensiva generale italiana. Assist Inferm Ric. 37(4): 181-188.

Minayo M. C. S (2014). O desafio do conhecimento: pesquisa qualitativa em saúde. $14^{\mathrm{a}}$ ed. São Paulo: Hucitec.

Minayo M. C. S (2017). Amostragem e saturação em pesquisa qualitativa: consensos e controvérsias. Revista Pesquisa Qualitativa. 5(7): 01-12.

Moré K. L. O. O (2015). A “entrevista em profundidade" ou "semiestruturada", no contexto da saúde. Dilemas epistemológicos e desafios de sua construção e aplicação. Investigação Qualitativa em Ciências Sociais. 3: 126-131.

National Pressure Ulcer Advisory Panel - Npuap. (2018). NPUAP pressure injury stages. Washington. Disponível em: http://www.npuap.org/resources/educational-andclinicalresources/npuap-pressure-injury-stages/. Acesso em: 07/05/2020. 
Neiva M. J. L. M et al (2019). Nursing care in prevention to pressure injuries in hospitalized patients. Brazilian Journal of Health Review. 2(5): 4336-4344.

Pereira A. S et al (2018). Metodologia da pesquisa científica. [e-book]. Santa Maria. Ed. UAB/NTE/UFSM. Acesso em: 24/05/20. Disponível em: https://repositorio.ufsm.br/bitstream/handle/1/15824/Lic_Computacao_MetodologiaPesquisa-Cientifica.pdf?sequence $=1$.

Riccioni $\mathrm{N}$ et al (2019). Interrater reliability of the Braden and Braden $\mathrm{Q}$ by skin champion nurses. Journal of pediatric nursing. 44: 9-15.

Rocha S. S et al (2020). Análise da presença de lesão por pressão em pacientes hospitalizados e as principais comorbidades associadas. Research, Society and Development. 9(4): e150943009.

Rodrigues A. S et al (2018). Lesão por pressão em paciente de unidade de terapia intensiva, características, causas, fatores de riscos e medidas preventivas. Revista Eletrônica Acervo Saúde. 10: S991-S996.

Rodríguez-Acelas A. L \& Cañon-Montañez W (2018). Contributions of health scales as tools that influence decisions in caring for patients. Revista Cuidarte. 9(1): 957-1960.

Saghaleini S. H et al (2018). Pressure ulcer and nutrition. Indian J Crit Care Med. 22(4): 28389.

Sanches B et al (2018). Adesão da enfermagem ao protocolo de lesão por pressão em unidade de terapia intensiva. Arquivos de Ciências da Saúde. 25(3): 27-31.

Serrano M. L et al (2017). Risco fatores para pressão úlcera desenvolvimento no Intensivo Cuidado Unidades: a sistemático reveja. Med Intensiva. 41(6): 339-346.

Silva D. R. A et al (2017). Pressure ulcer dressings in critical patients: a cost analysis. Rev Esc Enferm USP. 51: e03231. 
Silva, F. D. V et al. (2019). Conhecimentos e práticas de enfermagem na prevenção e cuidado às lesões por pressão. Revista de Enfermagem da UFSM. 9(4): 1-16.

Silva G. M. M et al (2014). Análise da mecânica respiratória nas posições decúbito dorsal, lateral e sentada, em pacientes em ventilação mecânica. Revista Coorte. 04: 31-38.

Sousa C. R et al (2017). Avaliação da incidência e prevalência de lesão por pressão em um hospital de urgência. Revista Uningá Review. 31(1):24-28.

Torres R. C et al (2018). Commission for Prevention and Treatment of Skin Injuries Implantation in a Public Hospital. Revista Enfermagem Atual InDerme. 86(24): 1-6.

Vasconcelos J. M. B \& Caliri M. H. L (2017). Nursing actions before and after a protocol for preventing pressure injury in intensive care. Escola Anna Nery. 21(1): 1-9.

\section{Porcentagem de contribuição de cada autor no manuscrito}

Luciane dos Santos Botelho - 34\%

Éder Luís Arboit - 33\%

Vera Lúcia Freitag - 33\% 